\title{
Brain changes associated with language development and learning: A primer on methodology and applications
}

Article

Accepted Version

Creative Commons: Attribution-Noncommercial-No Derivative Works 4.0

Luk, G., Pliatsikas, C. and Rossi, E. (2020) Brain changes associated with language development and learning: A primer on methodology and applications. System, 89. 102209. ISSN 0346-251X doi: https://doi.org/10.1016/j.system.2020.102209 Available at https://centaur.reading.ac.uk/88597/

It is advisable to refer to the publisher's version if you intend to cite from the work. See Guidance on citing.

To link to this article DOI: http://dx.doi.org/10.1016/j.system.2020.102209

Publisher: Elsevier

All outputs in CentAUR are protected by Intellectual Property Rights law, including copyright law. Copyright and IPR is retained by the creators or other copyright holders. Terms and conditions for use of this material are defined in the End User Agreement.

www.reading.ac.uk/centaur 
Central Archive at the University of Reading

Reading's research outputs online 


\section{AUTHORS ACCEPTED VERSION}

To appear in System

Brain changes associated with language development and learning: A primer on methodology and applications

Gigi Luk ${ }^{1}$, Christos Pliatsikas ${ }^{2,3}$, Eleonora Rossi ${ }^{4}$

1. Department of Educational and Counselling Psychology, McGill University, Canada

2. School of Psychology and Clinical Language Sciences, University of Reading, UK

3. Facultad de Lenguas y Educación, Universidad Nebrija, Spain

4. Linguistics Department, University of Florida, USA 


\begin{abstract}
Brain plasticity associated with second language acquisition and learning has been a focus of research in the past two decades. Recent research on cognitive neuroscience has enriched current understanding on the neurological underpinning of second language learning. Beyond behavioral findings, examining brain functions and structures provides a biological explanation of how language acquisition (as a natural experience) and learning (as an active skill and knowledge acquisition process) shapes the human brain. Together, combining cognitive neuroscience methods and second language acquisition and learning has offered an opportunity for cross-disciplinary collaboration. To facilitate cross-disciplinary understanding and potential research collaboration, this review paper aims to provide an overview of the major cognitive neuroscience methodologies adopted to study second language acquisition and learning. A selection of empirical studies covers second language acquisition in developing children, bilingualism as a naturally-occurring experience, and short-term second language learning in laboratory settings. Brain structural (diffusion tensor imaging, DTI; and voxel-based morphometry, VBM) and functional (electroencephalography, EEG; and eventrelated potentials, EPRs) methods are briefly discussed with suggested further readings. The paper ends with future directions using these methodologies to explore brain changes in response to second language teaching and learning experience.
\end{abstract}

Keywords: bilingualism, cognitive neuroscience, second language learning, structural MRI, EEG/ERPs 
Brain changes associated with language development and learning:

A primer on methodology and applications

\section{Introduction}

Recent scientific advances have enabled scientists to examine brain changes in relation to life experiences associated with language use. Of particular interest is how human adapts to the demand of acquiring two (or more) languages naturally (Bialystok, 2017), but less is known about how foreign language learning and teaching differs from naturalistic immersion experience. Naturalistic immersion experience in a second language has been examined in the field of cognitive neuroscience using a variety of methods, such as functional and structural magnetic resonance imaging (MRI, e.g., Silva \& Clitterio, 2017), diffusion tensor imaging (DTI, e.g., Rollans, Cheema, Georgiou, \& Cummine, 2017), electroencephalography (EEG) and Event-Related Potentials (ERPs) (for a recent review, see Beres, 2017), functional nearinfrared spectroscopy (fNIRS) (e.g., Wan, Hancock, Moon, \& Gillam, 2018; for a general review, see Pinti, Tachtsidis I, Hamilton, Hirsch, Aichelburg, et al., 2018), and magnetoencephalography (MEG) (e.g., Brodbeck \& Pylkkänen, 2017).

As expected, each of these methods has its strengths and weaknesses. One common strength is that all the methods provide insights into how brain functions while processing language. However, only structural MRI and DTI allow researchers to examine brain structure, grey and white matter respectively, in relation to language learning. In this review, we provide an overview of the methods associated with understanding brain functions and structures in psycholinguistics, particularly in bilingualism and foreign language acquisition. The overview is expected to provide general information about these methods to facilitate research consumption as well as cross-disciplinary research collaboration combining psycholinguistics and cognitive neuroscience.

1.1 Goal of the present review 
The present review aims to connect cognitive neuroscience and psycholinguistic research through the topics of second language (L2) acquisition and learning. This interdisciplinary collaboration is essential and timely. The proportion of bilingual individuals is currently on the rise in North America and Europe (Eurostat, 2019; Statistics Canada, 2019; Ryan, 2013). While significant advances have been made in understanding second language acquisition and learning, the knowledge has mostly been focused on behavioral outcomes and in naturalistic L2 immersion experience. In cognitive neuroscience, how the brain handles language processing is still largely based on monolinguals. Understanding the neural mechanisms and dynamics involved in managing multiple languages is necessary to provide a diverse and realistic view of language processing for many. Therefore, joining forces between cognitive neuroscientists and applied linguists on second language acquisition and learning is a fruitful collaboration introducing linguistic diversity into cognitive neuroscience research. The purpose of this review is to facilitate the understanding of the research methodology in cognitive neuroscience and encourage the cross-disciplinary collaboration to fill the knowledge gap on brain responses to specific pedagogical focuses. We begin by providing an overview of the research approaches in MRI and EEG, followed by applications of these methodologies in studying second language acquisition.

1.2 Research approaches to study the human brain

The human brain is composed of grey matter, white matter, and cerebral spinal fluid. The high-resolution structural image allows the visual inspection these three elements (see Figure 1). Grey matter is largely composed of neurons, while white matter forms pathways, including axons and glia cells, that connect neurons in different regions of the brain (Edgar \& Griffiths, 2009; for an overview video on neuroanatomy, please see JoVE Science Education Database, 2020: https://www.jove.com/science-education/5204/an-introduction-toneuroanatomy). White matter pathways are wrapped with myelin sheath which is made up of 
fatty tissues that act as a barrier wrapping the axons. Diffusion Tensor Imaging (DTI) allows researchers to examine microscopic structure of white matter pathways by analysing diffusion of water molecules. DTI extracts information about water diffusion within the white matter pathways. Another brain structure analysis technique focuses on the grey matter. Using Voxel-Based Morphormetry (VBM), researchers segmented grey matter from neighboring white matter. After the segmentation, the amount of grey matter can be quantified in each voxel and provide an estimate of grey matter in different brain regions. These quantities can be correlated with language performance or compared between groups with different language experiences (e.g., Pliatsikas, Johnstone, \& Marinis, 2014).

<Insert Figure 1 about here>

To understand neurolinguistic processes, an approach is needed to capture brain function at a time scale that simulates language processing. One such approach is to examine the electrical signal of the brain that is generated by changes in action potentials in neural (cortical/pyramidal) cells. This signal is very fast, with action potentials occurring as quickly as one millisecond, and reaching firing rates of hundreds of events per second (Buzsáki, 2006). As such, EEG is best suited to study the cortical brain activity underlying cognitive functions with a fast and accurate temporal resolution, but not necessarily identifying where the source of the signal comes from.

2. MRI approaches to examine brain structural differences and language processing

Using MRI approaches, high resolution images of white matter and grey matter can be captured using DTI and VBM respectively. Data analysis of MRI data is typically done on a computing cluster rather than an individual computer as the analysis are often computationally intensive. There are several software packages available for the preprocessing and analysis of DTI data (see Soares et al., 2013, for a list). In this review, we refer to the tools included in the FSL package (Jenkinson, Beckmann, Behrens, Woolrich, \& 
Smith, 2012). FSL is an open access software library for the analysis of most types of MRI data. It is free to install and use, it runs on a Linux environment, including most of the more common distributions, or on Windows via a virtual machine, and it requires basic knowledge of shell scripting. More details, including installation instructions, tutorials and sample data, can be found at the FMRIB software library (Analysis Group at the Wellcome Center for Integrative Imaging, 2018, https://fsl.fmrib.ox.ac.uk/fsl/). In this section, an overview of the methods underlying DTI and VBM is described, followed by a discussion of the applications of DTI and VBM in applied linguistics.

\subsection{Diffusion Tensor Imaging}

Diffusion tensor imaging is a MR technique that capture directional water displacements in white matter tracts (Bihan, Mangin, Poupon, Clark, Pappata, et al., 2001). DTI data is collected in a MR scanner using a different sequence. The duration of the scan ranges from 4 minutes to 10 minutes, depending on the resolution and scanner characteristics. DTI data allow researchers to estimate water diffusivity in each voxel of the brain. A voxel is a three-dimensional space represented as a unit in the brain after adjusting to a standard template. A model describing the three-dimensional diffusivity direction is called a tensor. In an unconstrained space, such as cerebrospinal fluid, water molecules diffuse in all directions in a random motion. This type of diffusivity is described as isotropic, i.e., equal towards all directions, forming a spherical tensor. In white matter tracts, however, myelin sheaths form water barrier and restricts water diffusivity, i.e., water molecules tend to diffuse along the shape of the white matter tracts. This type of diffusivity behaviour is called anisotropic, forming an elongated tensor mirroring the axons along the white matter tract.

The tensor model in each voxel allows the calculation of diffusivity and anisotropy to describe the shape of the white matter tract with water displacement as a proxy. Common 
measures of diffusivity and anisotropy are 1) Axial diffusivity (AD), indicating diffusivity parallel to a white matter tract; 2) Radial diffusivity (RD), indicating the diffusivity of water molecules perpendicular to a white matter tract; 3) Mean diffusivity (MD), representing the average diffusivity across all directions; and finally 4) Fractional anisotropy (FA), indexing whether the diffusion is isotropic (when the value is 0 , indicating non-directional diffusion) or anisotropic (when the value is 1 , indicating complete directional diffusion). Figure $2 \mathrm{a}$ shows an image of the diffusion directions in each voxel in the corpus callosum. Corpus callosum is the white matter pathway that connects the left and right hemispheres. Since there are few crossing fibres in the corpus callosum, the lines reveal that there is a high level of directional diffusion in this white matter pathway (an example of anisotropic diffusion). In contrary, in Figure 2b, there are crossing fibres in the sagittal stratum which includes the left inferior fronto-occipital fasciculus and left inferior longitudinal fasciculus. When the two pathways cross, the FA values in this region decreases because of the different directions of the pathways. Therefore, interpretation of FA values should be combined with familiarity of anatomical structure of the white matter architecture.

$<$ Insert Figure 2 about here>

\subsection{Voxel-Based Morphometry}

In addition to examination of white matter, recent research has also investigated grey matter in the brain. The term grey matter refers to the cell bodies of the brain neurons which are found concentrated on the surface of the cerebral and the cerebellar cortex, as well as in nuclei deep in the brain underneath the white matter. These include the putamen, caudate nucleus, globus pallidus and other smaller structures, collectively called the basal ganglia, as well as the thalamus. One of the most commonly used methods for the study of grey matter is Voxel-Based Morphometry (VBM, Good et al., 2001). VBM is an unbiased whole brain method which allows for the segmentation and quantification of grey matter. The results of the 
quantification can be used in subsequent comparisons between groups or as a predicted value in correlational designs. Indeed, VBM is an analytic technique that can also be applied to white matter analysis.

Another method that is gaining prominence in the literature is the study of the shape of subcortical structures via a vertex-based surface analysis, implemented in FSL as FIRST (Patenaude, Smith, Kennedy, \& Jenkinson, 2011). FIRST provides standard templates of the main subcortical structures and estimates the degree and direction of surface displacement for each of the tested participants. These estimates can subsequently be compared at the group level or used in correlational designs, similar to the estimations provided by VBM. Additionally, FIRST can provide measures of volume for each of the subcortical structures, which can be used in similar ways (for example uses of FIRST, see Burgaleta et al., 2016; Pliatsikas, DeLuca, Moschopoulou, \& Saddy, 2017). The added benefit of using FIRST instead of/in addition to VBM is that the small volume of the subcortical structures might mean that any effects found in them may not survive the whole-brain correction for multiple comparisons as implemented in VBM; moreover, VBM has no way of accounting for subcortical reshaping that might not translate into volumetric changes. Researchers that would consider using both methods with the same samples are advised to exclude from the VBM analysis the subcortical structures that they will include in the FIRST analysis.

\subsection{Applications of DTI and VBM in understanding language development}

Human brain plasticity in the white matter pathways have been studied in many different domains (for a review, please see Sampaio-Baptista \& Johansen-Berg, 2017). With language experience being one of the most persistent and prevalent experience, researchers began researching white matter pathways that are crucial for language processing. In this section, 
we provide an overview of empirical evidence connecting white matter plasticity and language development, language experience, and language training.

\subsubsection{White matter pathways changes associated with language development}

To appreciate the how the human brain changes along with language development, researchers examined the neural pathways responsive to human language in the earliest stage of life. Perani and colleagues (2011) reported that one to three days old infants showed functional connectivity between the bilateral temporal regions when responding to human speech, whereas adults showed more intrahemispheric connectivity between frontal and temporal regions in the left hemisphere. This finding illustrates that there is biological readiness for newborns to be responsive to language, with white matter architecture supporting the functional regions responsible for language acquisition. A subsequent followup study further confirmed that the immature pathway in newborns is developing in school age children, which shows more developed pathways than newborns, but still immature relative to that observed in the adults (Brauer, Anwander, Perani \& Friederici, 2013).

Skeide, Brauer, and Friederici (2016) continued to examine the relationship between white matter pathways development and functional neural network when children and adults were listening to syntactically simple and complex sentences. Using a cross-sectional design, children from the ages of three to ten and adults performed a sentence-picture matching task while undergoing fMRI scans. Results showed older children responded faster and more accurate than younger children, with overall performance level approaching adults.

Importantly, the same pattern of results was observed in the variation of fractional anisotropy in the two white matter pathways connecting the frontal and temporal regions of the brain, namely the arcuate fasciculus. Subsequent research on a large sample of children $(N=183)$ from three months to four years also reported the volume fraction in myelination in major white matter pathways, such as bilateral corona radiata, pathways in the left inferior frontal 
regions, and anterior corpus callosum, are significantly correlated with age and expressive and receptive vocabulary (O'Muircheartaigh, Dean III, Ginestet, Walker, Waskiewicz, et al., 2014).

\subsubsection{Individual differences in language experience}

In addition to typical and atypical language development, the investigation on white matter pathways has also been focusing on individual differences in language acquisition, such as bilingualism. Bilingualism refers to the use of two (or more) languages on a daily basis (Grosjean, 2013). For many bilinguals, using both languages daily is part of their life and the experience is persistent. This presents an opportunity for researchers to employ neuroimaging techniques to investigate brain functions and structures associated with natural everyday experience. Notably, like many life experiences, bilingualism is complex and dynamic. This complexity has been a challenge in research as researchers around the world are conducting work on bilingualism, the operational definitions of bilinguals (and sometimes monolinguals) may not be consistent (Surrain \& Luk, 2019). This is understandable given bilingualism is an interactional experience between an individual and his/her environment. This complexity is especially relevant for neuroimaging researchers. Given the high cost of the research, many studies adopt group comparisons to achieve an efficient design. As such, it is crucial for researchers to report individuals' experience as a group as well as the sociolinguistic environment for readers to understand the different language interactions in the groups.

The investigation of brain plasticity associated with bilingualism has largely been on adult and the aging populations, with much limited research on developing samples (see Pliatsikas, 2019, for a review of the available evidence). Luk, Bialystok, Craik \& Grady (2011) has shown that lifelong bilingual older adults had higher FA in corpus callosum extending to the right inferior frontal regions. This finding was also reported in young adults 
in another study (Pliatsikas, Moschopoulou \& Saddy, 2015). Findings in these two studies contradict other studies in which monolingual young adults (Cummine \& Boliek, 2013; Kuhl, Stevenson, Corrigan, van den Bosch, Can \& Richards, 2016) and older adults (Gold, Johnson, \& Powell, 2013) showed no difference in FA or higher FA in selected regions respectively. Gold et al. (2013) has pointed out that the bilingual participants in the study reported higher incidence of preclinical Alzheimer's Disease whereas the participants in Luk et al. (2011) study did not have these symptoms. Recently, Anderson, Grundy, De Frutos, Barker, Grady \& Bialystok (2018) revisited the paradoxical results and reported that when participants were matched using propensity scores in their groupings and statistically control for other covariates, bilingual older adults were reported to have higher axial diffusivity in the left superior longitudinal fasciculus. Axial diffusivity represents the rate of diffusion (Feldman, Yeatman, Lee, Barde, \& Gaman-Bean, 2010) whereas fractional anisotropy is a summary measure of estimate between isotrophy and anisotropy of diffusion. Collectively, results showed that there are noticeable differences in white matter architecture between monolinguals and bilinguals.

Researchers have also compared differences in white matter pathways in bilinguals with different experiences. Generally, three dimensions define bilinguals' experiences: onset age of second language (L2) acquisition, second language proficiency, and frequency or usage of two or more languages (Luk et al., 2011, 2013). If bilingualism is considered as an experience-dependent mechanism, then onset age of L2 acquisition is an important variable because brain plasticity peaks during childhood and gradually declines (for a general review, see Power \& Schlagger, 2018; for a review specifically related to bilingualism, see Berken, Gracco \& Klein, 2016). Young adults who acquired L2 early in life (before 5) had qualitatively different white matter structures compared to young adults who acquired L2 later in life (around 9; Hämäläinen, Sairanen, Leminen, \& Lehtonen, 2017), although the 
differences were observed in different tracts with different metrics: Early bilinguals had higher FA in the anterior region of the left arcuate fasciculus but higher MD in bilateral inferior fronto-occipital fasciculi. The nature of these findings suggests the timing of L2 acquisition is important to consider when investigating bilingualism as a contributing factor to brain structural plasticity. While these findings concern naturalistic bilingual experience, it implicates the sensitive period during which the human brain is most responsive to L2 usage and experience.

While group comparisons are informative, group categorization is usually relative within a sample and therefore, may not be reproducible in samples with different quality of bilingualism. For example, the groups form in communities with a strong dominant language is not the same as the groups form in multilingual communities. Therefore, studies using a single sample of bilingual correlating dimensions of bilingualism and white matter pathways. Nichols and Joanisse (2016) reported that age of L2 acquisition (English in this case) and L2 proficiency differentially predicted variation in the white matter pathways in the left (corpus callosum, arcuate fasciculus, and inferior longitudinal fasciculus) and right (inferior longitudinal fasciculus, arcuate fasciculus, forceps minor) hemispheres respectively. The participants were a group of Chinese students studying in Canada who have not been fully immersed in the English-speaking environment at the time of testing. To examine the role of immersion, Kuhl, Stevenson, Corrigan, van den Bosch, Can, and Richards (2016) reported that duration of residence was significantly correlated with distributed white matter pathways in bilingual Spanish-English speakers living in the U.S. such that the longer an individual stayed in an L2 immersive environment, the higher FA (and lower diffusivity) was observed in white matter pathways in both left and right hemispheres. While the duration of residence may not imply L2 acquisition, Pliatsikas and colleagues (2017) compared individuals who were dominant English speakers but learned a second language at different point in 
childhood, significant correlations between onset age of L2 acquisition and bilateral white matter pathways were reported. Similarly, Pliatsikas (2019) reported a correlation between the age of L2 acquisition and fractional anisotropy in the corpus callosum in a sample of immersed bilinguals. These findings echoed those reported in Kuhl et al. (2016) and highlights the relationship between L2 acquisition and changes in white matter pathways.

\subsubsection{Longitudinal changes in white matter pathways after language learning}

The strongest evidence supporting brain plasticity associated with language acquisition comes from longitudinal studies. However, due to the high cost and logistical challenges, there are few neuroimaging studies on white matter pathways changes following naturalistic language acquisition or structured language learning. Mohades and colleagues (2012, 2015) had reported in two studies following a group of children longitudinally. In their 2012 study, the research team compared simultaneous bilinguals, sequential bilinguals, and monolinguals who were between the ages of 8 and 11 on four white matter pathways: left arcuate fasiculus, left inferior occipitofrontal fasciculus, anterior corpus callosum projecting to orbital lobe, and the anterior midbody of corpus callosum projecting to premotor and supplementary motor area. Results showed that simultaneous bilinguals had higher FA than monolingual children in the left inferior occipitofrontal fasciculus while the monolinguals had higher FA in the anterior corpus callosum projecting to orbital lobe. Sequential bilinguals had FA values in between the simultaneous bilinguals and the monolinguals, but the values were not statistically different from either group. Two years later, the children were being assessed again. Simultaneous bilinguals had the highest level of FA in the left inferior occipitofrontal fasciculus. However, in regard to the rate of change, sequential bilinguals demonstrated the largest level of growth compared to the other groups. These findings showed that onset age of second language acquisition is associated with white matter plasticity in developing samples. 


\section{EEG/ERPs}

MRI research findings are most sensitive to long-term persistent language experience. To evaluate brain responses closer to real-time processing, such as learning vocabulary in a new language, researchers rely on the electroencephalogram (EEG). The field of brain imaging has had a long tradition of analysing the spontaneous electrical activity of the brain to unveil human cognitive functions, including language. A century of electrophysiological research has demonstrated that neural oscillations represent the spontaneous, endogenous functioning of the brain (Buzsáki et al., 2012). Since Berger's initial observations correlation slow oscillatory brain activity to rest (i.e., alpha waves), the human EEG has been one of the primary sources of information to research the neural mechanisms underlying human cognition.

\subsection{EEG and Event Related Potentials: a methodological introduction}

Continuous EEG activity provides a rich source of data that can be analyzed in a number of ways. Traditionally, researchers have relied on analyzing the continuous EEG signal locked to a specific experimental stimulus. After averaging across many trials presenting similar experimental stimuli in relation to baseline, Event Related Potentials (ERPs) can be measured across many participants. By having a sufficiently large number of trials for each experimental condition and with a large enough sample, the signal to noise ratio increases, and the time course of activation evoked by the stimulus can be tracked with a good amount of precision, resulting in reliable and analyzable ERPs. Decades of research using ERPs for language research have revealed that there are a number of ERP components that are specific to a number of language processes. In the next section, we will introduce some basic descriptions of how task-related ERP data is collected and analysed, and we will also provide some examples to describe major ERP components such as the P600 (appears around $600 \mathrm{~ms}$ 
after the stimulus presentation) and the N400 (appears around 400ms after stimulus presentation) that have been shown to be foundational also when understanding changes to the native language related to second language processing (Bice \& Kroll, 2015). We chose to focus on these two components, namely the P600 and N400, due to their robust empirical relationship to morpho-syntactic and semantic processing respectively (for a description of additional ERP components, please see Kappenman \& Luck, 2011).

When analyzing the EEG signal locked to a specific experimental manipulation (see below for details on experimental paradigms and analysis), all the residual cognitive activity not time-locked to the onset of the experimental stimuli is lost after averaging, leaving only meaningful signal relevant to processing of the experimental stimuli. In order to capture variability in the EEG signal that might be lost after averaging, scientists have recently started to decompose the EEG signal into different frequencies, and investigate the local (the amount of power in each frequency band), and long-range synchrony (oscillatory phase relation measures between different sources/channels) of those frequencies to help capturing the dynamics of task that are independent of linguistic processes, as well as domain-general cognitive processes (i.e., memory encoding and retrieval, attentional mechanisms) associated with language comprehension and production (i.e., Rossi \& Prystauka, in press). Understanding the oscillatory brain activity during complex cognitive (linguistic) tasks enables researchers to highlight individual linguistic and cognitive components that might not be possible to distinguish when analyzing the signal in the time domain (i.e., ERPs). The range of brain oscillatory signal is usually subdivided into 5 frequency bands: delta $(1-3 \mathrm{~Hz})$, theta $(4-7 \mathrm{~Hz})$, alpha $(8-12 \mathrm{~Hz})$, beta $(13-30 \mathrm{~Hz})$ and gamma $(>31 \mathrm{~Hz})$. Specifically, a number of frequencies have been highlighted as important for a number of linguistic processes. For example, theta activity is important for lexical retrieval, alpha and beta are engaged during semantic and syntactic unification, and gamma activity has been connected to prediction 
during sentence processing (Lam, Schoffelen, Uddén, Hultén and Hagoort, 2016). In addition, alpha has been consistently related to increased attentional demands (Klimesch, 2012). For recent excellent reviews on language related oscillatory components, we refer the reader to Prystauka and Lewis (2019), Murphy et al. (2019) and Meyer (2017).

\subsection{ERPs, Time-Frequency Representation analysis, and Resting State EEG: An} introduction

Most of the neurophysiological findings in the language sciences have focused on the analysis of the continuous EEG signal locked to specific stimuli, and primarily by describing the signal in the time-domain (i.e., Event-Related Potentials). The analysis and comparison of the averaged EEG signal in a specific point in time for each experimental condition results in ERP components that are usually characterized in terms of temporal resolution, amplitude, and scalp distribution. In order to extract the EEG signal locked to specific experimental conditions, scientists rely on sending specific time markers (triggers) during the continuous EEG signal that are necessary to track at what exact point in time a stimulus occurred and are crucial to analyze changes of the EEG signal occurring at that specific point. For example, in order to study the on-line brain response to verb processing, sentences with a correct verb such as: "The scientist eats an apple" will be compared to sentences with a verb violation such as: "The scientist *eat an apple". Critically, two different trigger codes will be sent locked to the beginning of the verb (i.,e, eat/eats). In this way, during the analysis it will be possible to extract the EEG signal in response to the correct verb and the incorrect verb, average the signal across a number of items, across a large enough participants sample, and compare the averaged ERP signal across the two conditions, i.e., ERP signature in response to the correct verb, and ERP signature in response to the incorrect verb. Under normal condition, ERP components will be revealed if there is a good signal to noise ratio. Besides 
overall good data quality, the more items involved in calculating the average per condition, the stronger the signal is relative to noise.

Decades of language-based ERP research have revealed that distinct linguistic processes elicit distinct neural responses. For example, lexico-semantic processes have been shown to be indexed primarily by a negative ongoing wave between 200 and $600 \mathrm{~ms}$ with maximum amplitude at around $400 \mathrm{~ms}$ after a stimulus occurs, the so-called N400 effect (Kutas \& Hillyard, 1980). For example, an N400 component is elicited when a sentence contains a semantic violation, such as: "The cat runs to the river and drinks some *wine". In the context of this sentence, the work wine is semantically infelicitous, and it elicits an N400 (Kutas and Federmeier, 2011, for a recent review on the N400). The N400 has also been examined in relation to a number of different aspects of L2 processing. For example, recent studies have shown that during a novel language learning paradigm, the brain reveals the emergence of an N400 even when there is no observable learning in behavior (McLaughlin, Osterhout, \& Kim, 2004; McLaughlin et al., 2010). In sum, the N400 is a very robust task induced neural component that emerges primarily for lexico-semantic processes.

The P600 component is instead a positive ongoing wave that emerges later than the N400, primarily between 400 and $900 \mathrm{~ms}$ with maximum amplitude around $600 \mathrm{~ms}$. The P600 (Osterhout \& Holcomb, 1992). The P600 is also task-related and is elicited by a number of morpho-syntactic violations, including violations of phrase-structure and subcategorization. For example, a P600 would be elicited by sentences such as: "The cat *run to the river and *drink some water" where the subject-verb inflection is incorrect. The P600 has generally been interpreted as signaling syntactic reanalysis and repair, and has also been linked to increasing integration difficulties and increased processing demands (Kaan \& Swaab, 2003). 
ERPs components result from averaging the EEG signal at a specific point in time when the EEG signal is analyzed in the time domain. However, when the EEG signal is averaged in the time domain, the frequency information portion of the signal is lost. Decomposing the EEG signal into different frequencies allows to capture the dynamics of language-specific and domain-general processes that are involved in language processing that might otherwise not be distinguishable (Bastiaansen, Mazaheri, \& Jensen, 2012). TimeFrequency Representation analysis (TFR), i.e., analyzing the properties of the EEG spectral signal in time, is a powerful analysis that enables to capture different subcomponents of complex cognitive processes that originate from the synchronization/desychronization of neuronal activity at certain frequencies, times, and neural locations. Crucially, TFR can highlight how distinct brain oscillations contribute to the formation of neuronal assemblies, which are thought to reflect different aspects of linguistic processing (Bastiaansen, Mazaheri, \& Jensen, 2012). In contrast to the time-locked analytic approach, TFR analyses are flexible because they also allow studying of the long-term oscillatory signal as language processing unfolds. This is more in line with how language is processed in reality. For example, Rossi \& Prystauka (in press), analyzed the TFR signal in response to pronoun processing in Spanish native speakers and L2 learners learning Spanish. In this specific study, the TFR analysis was locked to the when the pronoun (correct or incorrect) appeared during sentence processing. The results revealed a modulation of alpha and beta frequencies in response to the violation. However, the added value of TFR analysis is that this can be used also to investigate the long-term synchrony of the oscillatory brain power, thus enabling to study the oscillatory behavior of the EEG signal as language unfolds (Prystauka \& Lewis, 2019). From an acquisition viewpoint, both ERPs and TFR rely on the same raw EEG signal. What distinguishes the two methods is the extraction of the signal in the time or in the frequency domain. 
More recently, psycho/neurolinguist have started to analyse the default electrical activity of the brain emerging in the absence of goal-directed behaviour. Different from ERPs and even frequency analysis of the EEG signal, the so-called resting-state oscillatory electrical signal (RS-EEG) of the brain has been highlighted as a crucial index of neural functioning (Buzsaki, 2006; Raichle et al., 2001) during wakeful rest and in the absence of any particular task-related event. RS-EEG reflects the intrinsic synchronism of the brain that likely represents an index of "readiness" of the neural system (Raichle \& Snyder, 2007). This notion is important for applied linguists as language production often begins with preparation before the actual production, and changes with cumulative experience, potentially shaping the intrinsic brain-base signal. Importantly, the RS-EEG signal is relatively stable across the lifespan and it does not easily change at shorter time frames (Anderson \& Perone, 2018). However, even though RS-EEG does not change as a reflection of fast cognitive performance, it is not a completely static, and slowly shifts during the lifespan, possibly reflecting neural reorganization and cognitive development throughout infancy, young adulthood and aging (Anderson \& Perone, 2018; Doppelmayer et al., 2002). In sum, RS-EEG can be considered a relatively stable "signature" of individuals' neural functioning that likely represents waxing and waning of the inner neural system. RS-EEG is relevant to the study of language processing and bilingualism, because it provides a window to understand how individual variation in one's neural "basic signature" can predict rates of new learning. Also, as we discuss later, understanding if a relatively stable neural signal such as RS-EEG can be temporarily molded by new language learning could provide data in support of the idea that the human neural system is more flexible than assumed.

3.3 ERPs, Time-Frequency Representation, and RS-EEG in psycholinguistic research

Over forty years electrophysiological language processing research have yielded an incredibly rich set of ERP data that have paved the way to foundational discoveries in the 
field. We have already mentioned how ERP components such as the N400 and the P600 have been critical to reveal neurally specific signatures in response to different linguistic processes. ERPs have been also critical to understand similarities and differences between native and second language processing even in speakers who acquire a second language past a critical period for language acquisition. To exemplify, Rossi, Kroll, \& Dussias (2014) analyzed ERPs to ask if adult English native speakers who were learners of Spanish as their second language could process grammatical gender and number marked on pronouns as native speakers'. Importantly, grammatical gender, that is gender marking for inanimate objects is a feature that is not present in English, but that is marked in Spanish, as for example in "Ana compra la manzanafeminine singular y $l a_{\text {feminine singular }}$ come. Crucially, for a referential expression to be correct in Spanish, pronouns following an antecedent need to match in gender and number. Because Grammatical gender is not present in English, the authors predicted that violations of grammatical gender marked on the pronoun would yield a P600 for native speakers, but would not show for second language learners. However, the authors also predicted that proficiency in the L2 would have modulated the effect such that highly proficient L2 speakers would show the emergence of a P600, similar to native speakers. The results supported the predictions. A violation of grammatical gender on the pronoun elicited a P600 in native Spanish speakers, and in highly proficient Spanish L2 speakers, but lower proficiency L2 speakers did not show any sensitivity. Many more examples could be described here. For a recent review on ERPs for L2 processing specifically we refer to Tolentino et al., 2011. This approach has potential to evaluate sensitivity to different semantic and syntactic violation following L2 learning.

Relatively few studies have researched the neural underpinnings of language processing by analyzing the EEG signal in the frequency domain. A particularly illustrative example is the study by Bakker et al. (2015) that investigated the changes in oscillatory 
dynamics during novel word learning. In that paradigm, participants were exposed to new words during a learning phase that were designed to compete with existing English words (i.e., cathedruk, competing with the existing word cathedral). In a second recall phase, participants were shown the newly learned words, and new unlearned words. EEG was collected and compared both during the learning and recall phase ( 24 hours later). As hypothesized, newly learned words showed longer RTs than unfamiliar new words at recall, suggesting that they started to compete for selection with existing lexical items (i.e., cathedruk competing for selection with cathedral). Crucially, the TFR results revealed an increase in theta frequencies $(4-8 \mathrm{~Hz})$ for the newly learned words which was interpreted as a marker of lexical access, and memory encoding.

Beyond the single word level, TFR research is critical to understand the neural mechanisms that are at the basis of sentence processing. For example, in a seminal study, Bastiaansen and Hagoort (2015) tested modulations of EEG power change for correct sentences, syntactically correct though meaningless sentences, or random word lists. The results revealed larger beta power $(16-21 \mathrm{~Hz})$ for syntactically correct sentences than for incorrect ones, whereas semantically incorrect sentences yielded an increase in gamma power, revealing that syntactic and semantic processing are possibly subserved by independent neural networks. These findings are supported by recent results for grammatical processing in second language speakers that show decrease in alpha and beta frequencies for sentences that contain an incorrect pronoun (Rossi \& Prystauka, in press). For extensive reviews on oscillatory mechanisms during word level and sentence processing see Meyer (2017) and Prystauka \& Lewis (2019).

Despite the large body RS-EEG research for generalized cognition, the use and analysis of RS-EEG in psycholinguistic research is still in its infancy. A very limited number of studies so far, have attempted to acquire and analyse individual RS-EEG to understand 
variability in second language learning. For example, in infancy, higher frontal gamma RSEEG power measured at critical language developmental milestones (16, 24 and 26 months) has been shown to predict productive language abilities at 4 and 5 years of age (Gou et al., 2001). The proposal is that higher gamma RS-EEG power during infancy modulates nonlinguistic cognitive functions such as attention and working memory that are key for successful language development. RS-EEG has recently also been reported to be a predictor of second language acquisition and aptitude past childhood.

Prat and colleagues (Prat et al., 2016) tested 16 English-speaking participants who completed eight week of French language training though an immersive computerized learning environment. RS-EEG was recorded prior to the beginning of the training, and was later correlated to the learning outcomes. The results revealed specifically low (13-14.5 Hz) and mid-beta $(15-17.5 \mathrm{~Hz}) \mathrm{RS}-\mathrm{EEG}$ frequencies were positively correlated with L2 learning rates, explaining up to $60 \%$ of the overall variance. The authors conclude that stable properties of the RS-EEG network at rest can be predictive of individual differences in new language learning, and language aptitude (especially beta frequencies). These first results were recently confirmed by another study by Prat and colleagues (Prat et al., 2018) in participants who completed 8 weeks of French instruction through a computerized language learning program. Once again, the results showed that higher beta power at the right hemisphere, and coherence measures in the right hemisphere predicted the rate of L2 learning, post-learning vocabulary retention, and variance in speech attempts. To date, the extant literature using RS-EEG to understand second language processing is limited to a few studies.

\section{Future directions and conclusion}


Human's ability of becoming bilingual affords an exciting research opportunity to examine brain plasticity and a persistent naturalistic life experience. One possible future research direction is to investigate how bilingualism and biliteracy independently relate to white matter plasticity and/or changes in EEG signal. Although it is widely believed that bilingualism and biliteracy are related, many bilinguals who reported using a non-dominant language are only capable of reading and writing in the community dominant language or the language of instruction. Neuroimaging research has shown that bilingualism modulates the white matter pathways relevant to reading (Bakhtiari, Boliek, \& Cummine, 2014) and that there are substantive differences in white matter pathways between typical and atypical readers during childhood (Christodoulou, Murtagh, Cyr, Perrachione, Chang, et al., 2017). However, it is still unclear how bilingualism and biliteracy separately modulates white matter pathways or ERPs in development. Importantly, there is little understanding in how bilingualism interacts with developmental disorders, such as reading difficulty. This investigation will shed light on the qualitative differences among bilinguals as well as enriching behavioural comparisons on typical and atypical reading development.

Another future research direction is to examine the relationship between foreign language ability and variation in white matter pathways. Recent research has demonstrated robust laterality in white matter pathways and ability to imitate foreign speech sounds (Vaquero, Rodríguez-Fornells, \& Reiterer, 2017), suggesting one source of the high variability in L2 speech production. Foreign language pronunciation has attracted growing interests in applied linguistics, particularly with the recent advancement in assessing foreign language pronunciation. Saito (2017) reported that measures for L2 pronunciation loaded on the same factor and this factor was only marginally correlated with sound-symbol correspondence, but not the other lexical measure in the LLAMA (Meara, 2005). Identifying the underlying neural network supporting different aspects of L2 acquisition will enhance 
further understanding of the process of language acquisition and inform developing effective pedagogies for L2 learning. Following the investigation L2 acquisition, future research could benefit from longitudinal designs that followed participants over time to examine changes in white matter pathways before and after learning a new language or as language proficiency increases in developmental samples.

The investigation of rapid changes in the EEG neural signal represent a very exciting opportunity to unveil how the neural system molds in response to new language learning, and bilingualism. For example, cutting edge analyses allow to understand how different spectral power variation happen in real time during short novel language training, revealing how different cognitive and linguistic mechanisms might interplay for successful learning (i.e., Kepinska et al., 2017; de Diego-Balaguer et al., 2011). In addition, correlating individual measures of the brain's EEG signature while at rest is emerging as a powerful tool to investigate variability in rate of learning and learning retention in time. As discussed, RSEEG is a stable signal that changes relatively slowly across the lifespan that reflects neural reshaping, and reorganization. As such, it could be hypothesized that despite its relative stability, RS-EEG could undergo faster, temporary changes as a consequence of novel and intensive new (language) learning. Observing rapid RS-EEG changes in response to new intensive language learning could suggest that the neural system is more malleable than previously thought even in the face of its relative stability. Despite the promises of using different modalities of EEGs to investigate brain changes in L2 learning, there is yet research examining the interaction between individual differences in learners, such as age or first language spoken, in response to how L2 is being taught. This potential collaboration could combine neuroscientific evidence along with behavioral outcomes in particular second language pedagogical framework. 
With all the promise about the cognitive neuroscience methods reviewed here, it should note that these methods are tools with maximized utility when combined with meaningful content knowledge. We see that the combination of second language acquisition/learning and cognitive neuroscience methods has tremendous research and practical potential to extend our current knowledge of brain-behavior relationship and the neural mechanism underlying language processing. Specifically, findings from both structural MRI and ERPs are most powerful when relating to either individual differences in experience accumulated over time or in at-the-moment online language or other cognitive processing.

Human brain's capacity to learn and adapt to her environment has providing exciting opportunities for researchers from multiple disciplines. Language use marks an important ability that is unique to human. With the recent advances of neuroimaging technique allowing researchers to investigate changes in the human brain, diffusion weighted imaging holds the promise to further our understanding on the human brain structure adapts to the linguistic environment. This paper serves as a primer for researchers who are interested in conducting neuroimaging research examining white matter pathways. The focus of this paper has been on analysis. It should note that many technicalities are involved in the data acquisition stage. Researchers interested in pursuing neuroimaging methods without prior experience are recommended to reach out to researchers or institutions that have existing infrastructures and personnel. Interdisciplinary collaboration enriches each participating field and collectively contributes to understanding language use and brain plasticity. 


\section{References}

Analysis Group at the Wellcome Centre for Integrative Imaging (2018). FMRIB Software Library v6.0. Retrieved from https://fsl.fmrib.ox.ac.uk/fsl/fslwiki/

Anderson, J. A. E., Grundy, J. G., De Frutos, J., Barker, R. M., Grady, C., \& Bialystok, E. (2018). Effects of bilingualism on white matter integrity in older adults. NeuroImage, 167, 143-150. http://doi.org/10.1016/j.neuroimage.2017.11.038

Anderson, A. J., \& Perone, S. (2018). Developmental change in the resting state electroencephalogram: Insights into cognition and the brain. Brain and Cognition, 126(January), 40-52. http://doi.org/10.1016/j.bandc.2018.08.001

Bakhtiari, R., Boliek, C., \& Cummine, J. (2014). Investigating the contribution of ventrallexical and dorsal-sublexical pathways during reading in bilinguals. Frontiers in Human Neuroscience, 8, 507. http://doi.org/10.3389/fnhum.2014.00507

Bakker, I., Takashima, A., van Hell, J. G., Janzen, G., \& McQueen, J. M. (2015). Changes in theta and beta oscillations as signatures of novel word consolidation. Journal of Cognitive Neuroscience, 27(7), 1286-1297. DOI: 10.1162/jocn_a_00801

Bastiaansen, M. \& Hagoort, P. (2015). Frequency-based segregation of syntactic and semantic unification during online sentence level language comprehension. Journal of Cognitive Neuroscience, 27, 2095-2107.

Bastiaansen, M. C. M., Mazaheri, A., \& Jensen, O. (2012). Beyond ERPs: oscillatory neuronal dynamics. In The Oxford handbook of event-related potential components (pp. 31-50). Oxford University Press. DOI:

10.1093/oxfordhb/9780195374148.013.0024

Beres, A. M. (2017). Time is of the essence: A review of electroencephalography (EEG) and event-related brain potentials (ERPs) in language research. Applied Psychophysiology and Biofeedback, 42(4), 247-255. http://doi.org/10.1007/s10484-017-9371-3 
Berken, J. A., Gracco, V. L., \& Klein, D. (2017). Early bilingualism, language attainment, and brain development. Neuropsychologia, 98(September 2016), 220-227. http://doi.org/10.1016/j.neuropsychologia.2016.08.031

Bialystok, E. (2017). The bilingual adaptation: How minds accommodate experience. Psychological Bulletin, 143(3), 233-262. http://doi.org/10.1037/bul0000099

Bice, K., \& Kroll, J. F. (2015). Native language change during early stages of second language learning. NeuroReport, 26(16), 966. doi: 10.1097/WNR.0000000000000453

Bihan, D. L., Mangin, J. F., Poupon, C., Clark, C. A., Pappata, S., Molko, N. \& Chabriat, H. (2001). Diffusion tensor imaging: Concepts and applications. Journal of Magnetic Resonance Imaging, 13(4), pp. 534-546.

Brauer, J., Anwander, A., Perani, D., \& Friederici, A. D. (2013). Dorsal and ventral pathways in language development. Brain and Language, 127(2), 289-295. http://doi.org/https://doi.org/10.1016/j.bandl.2013.03.001

Brodbeck, C., \& Pylkkänen, L. (2017). Language in context: Characterizing the comprehension of referential expressions with MEG. NeuroImage, 147, 447-460. http://doi.org/https://doi.org/10.1016/j.neuroimage.2016.12.006

Burgaleta M., Sanjuán A., Ventura-Campos N., Sebastián-Gallés N., \& Ávila C. (2016) Bilingualism at the core of the brain. Structural differences between bilinguals and monolinguals revealed by subcortical shape analysis. Neuroimage, 125, 437-445.

Buzsáki, G. (2006). Rhythms of the Brain. New York, NY: Oxford University Press.

Buzsáki, G., Anastassiou, C. A. \& Koch, C. (2012). The origin of extracellular fields and currents -- EEG, ECoG, LFP and Spikes. Nature Reviews Neuroscience, 13(6), pp. 407-420.

Christodoulou, J. A., Murtagh, J., Cyr, A., Perrachione, T. K., Chang, P., Halverson, K., ... Gabrieli, J. D. E. (2017). Relation of white-matter microstructure to reading ability 
and disability in beginning readers. Neuropsychology, 31(5), 508-515. http://doi.org/10.1037/neu0000243

Cummine, J., \& Boliek, C. A. (2013). Understanding white matter integrity stability for bilinguals on language status and reading performance. Brain Structure and Function, 218(2), 595-601. Retrieved from https://doi.org/10.1007/s00429-012-0466-6

de Diego-Balaguer, R., Fuentemilla, L., \& Rodriguez-Fornells, A. (2011). Brain dynamics sustaining rapid rule extraction from speech. Journal of Cognitive Neuroscience, 23(10), 3105-3120. https://doi.org/10.1162/jocn.2011.21636

Doppelmayr, M., Klimesch, W., Stadler, W., Pöllhuber, D., \& Heine, C. (2002). EEG alpha power and intelligence. Intelligence, 30(3), 289-302. https://doi.org/10.1016/s0160-

\section{$\underline{2896(01) 00101-5}$}

Edgar, J. M., \& Griffiths, I. R. (2009). Chapter 5 - White matter structure: A microscopist's view. In H. Johansen-Berg \& T. E. J. B. T.-D. M. R. I. Behrens (Eds.), Diffusion MRI: From quantitative measurement to in vivo neuroanatomy (pp. 74-103). San Diego: Academic Press. http://doi.org/https://doi.org/10.1016/B978-0-12-374709-9.00005-5

Eurostat (April, 2019). Foreign language skills statistics. Retrieved from https://ec.europa.eu/eurostat/statisticsexplained/index.php/Foreign_language_skills_statistics

Feldman, H. M., Yeatman, J. D., Lee, E. S., Barde, L. H. F., \& Gaman-Bean, S. (2010). Diffusion tensor imaging: A review for pediatric researchers and clinicians. Journal of Developmental and Behavioral Pediatrics, 31(4), 346-356. http://doi.org/10.1097/DBP.0b013e3181dcaa8b

Gold, B. T., Johnson, N. F., \& Powell, D. K. (2013). Lifelong bilingualism contributes to cognitive reserve against white matter integrity declines in aging. Neuropsychologia, 51(13), 2841-2846. 
http://doi.org/https://doi.org/10.1016/j.neuropsychologia.2013.09.037

Good, C. D., Johnsrude, I. S., Ashburner, J., Henson, R. N., Friston, K. J. \& Frackowiak, R. S. (2001). A voxel-based morphometric study of ageing in 465 normal adult human brains. NeuroImage, 14 (1 Pt 1), pp. 21-36.

Grosjean, F. (2013). Bilingualism: A short introduction. In F. Grosjean, \& P. Li (Eds.), The psycholinguistics of bilingualism (2nd ed., pp. 5-25). Hoboken, NJ: Wiley-Blackwell.

Hämäläinen, S., Sairanen, V., Leminen, A., \& Lehtonen, M. (2017). Bilingualism modulates the white matter structure of language-related pathways. NeuroImage, 152, 249-257. http://doi.org/https://doi.org/10.1016/j.neuroimage.2017.02.081

Jenkinson, M., Beckmann, C. F., Behrens, T. E. J., Woolrich, M. W., \& Smith, S. M. (2012). FSL. NeuroImage, 62(2), 782-790.

http://doi.org/https://doi.org/10.1016/j.neuroimage.2011.09.015

JoVE Science Education Database. Neuroscience. An Introduction to Neuroanatomy. JoVE, Cambridge, MA, (2019). https://www.jove.com/science-education/5204/anintroduction-to-neuroanatomy

Kaan, E., \& Swaab, T. Y. (2003). Repair, revision, and complexity in syntactic analysis: An electrophysiological differentiation. Journal of cognitive neuroscience, 15(1), 98-110. DOI: $10.1162 / 089892903321107855$

Kappenman, E. S., \& Luck, S. J. (Eds.). (2011). The Oxford Handbook of Event-Related Potential Components. Oxford university Press. https://doi.org/10.1093/oxfordhb/9780195374148.001.0001

Kepinska, O., Pereda, E., Caspers, J., \& Schiller, N. O. (2017). Neural oscillatory mechanisms during novel grammar learning underlying language analytical abilities. Brain and language, 175, 99-110. https://doi.org/10.1016/j.bandl.2017.10.003 
Klimesch, W. (2012). $\alpha$-band oscillations, attention, and controlled access to stored information. Trends in Cognitive Science, 16(12), 606-617.

Kuhl, P. K., Stevenson, J., Corrigan, N. M., van den Bosch, J. J. F., Can, D. D., \& Richards, T. (2016). Neuroimaging of the bilingual brain: Structural brain correlates of listening and speaking in a second language. Brain and Language, 162, 1-9. http://doi.org/https://doi.org/10.1016/j.bandl.2016.07.004

Kutas, M., \& Federmeier, K. D. (2011). Thirty years and counting: Finding meaning in the N400 component of the event-related brain potential (ERP). Annual Review of Psychology, 62, 621-647.

Kutas, M., \& Hillyard, S. A. (1980). Event-related brain potentials to semantically inappropriate and surprisingly large words. Biological psychology, 11(2), 99-116. DOI: 10.1016/0301-0511(80)90046-0

Lam, N. H. L., Schoffelen, J.-M., Uddén, J. Hultén, A. \& Hagoort, P. (2016). Neural activity during sentence processing as reflected in theta, alpha, beta, and gamma oscillations. NeuroImage, 142, pp. 43-54.

Luk, G., Bialystok, E., Craik, F. I. M., \& Grady, C. L. (2011). Lifelong bilingualism maintains white matter integrity in older adults. The Journal of Neuroscience: The Official Journal of the Society for Neuroscience, 31(46), 16808-16813. http://doi.org/10.1523/JNEUROSCI.4563-11.2011

Luk, G., De Sa, E., \& Bialystok, E. (2011). Is there a relation between onset age of bilingualism and enhancement of cognitive control? Bilingualism: Language and Cognition, 14(4), 588-595. http://doi.org/DOI: 10.1017/S1366728911000010

McLaughlin, J., Osterhout, L., \& Kim, A. (2004). Neural correlates of second-language word learning: Minimal instruction produces rapid change. Nature neuroscience, 7(7), 703. DOI: $10.1038 / \mathrm{nn} 1264$ 
McLaughlin, J., Tanner, D., Pitkänen, I., Frenck-Mestre, C., Inoue, K., Valentine, G., \& Osterhout, L. (2010). Brain potentials reveal discrete stages of L2 grammatical learning. Language Learning, 60, 123-150. https://doi.org/10.1111/j.14679922.2010.00604.x

Meara, P. (2005). LLAMA Language Aptitude Tests: The Manual. Swansea: Lognostics. (Tech. Rep.). Retrieved from http://www.lognostics.co.uk/tools/index.htm

Meyer, L. (2017). The neural oscillations of speech processing and language comprehension: state of the art and emerging mechanisms. European Journal of Neuroscience, 48(7), 2609-2621. DOI: 10.1111/ejn.13748

Mohades, S. G., Struys, E., Van Schuerbeek, P., Mondt, K., Van De Craen, P., \& Luypaert, R. (2012). DTI reveals structural differences in white matter tracts between bilingual and monolingual children. Brain Research, 1435, 72-80. http://doi.org/https://doi.org/10.1016/j.brainres.2011.12.005

Mohades, S. G., Van Schuerbeek, P., Rosseel, Y., Van De Craen, P., Luypaert, R., \& Baeken, C. (2015). White-matter development is different in bilingual and monolingual children: A longitudinal DTI study. PloS One, 10(2), e0117968. http://doi.org/10.1371/journal.pone.0117968

Nichols, E. S., \& Joanisse, M. F. (2016). Functional activity and white matter microstructure reveal the independent effects of age of acquisition and proficiency on secondlanguage learning. NeuroImage, 143, 15-25. http://doi.org/https://doi.org/10.1016/j.neuroimage.2016.08.053

O’Muircheartaigh, J., Dean III, D. C., Ginestet, C. E., Walker, L., Waskiewicz, N., Lehman, K., ... Deoni, S. C. (2014). White matter development and early cognition in babies and toddlers. Human Brain Mapping, 35(9), 4475-4487. http://doi.org/10.1002/hbm.22488 
Osterhout, L., \& Holcomb, P. (1992). Event-related brain potentials elicited by syntactic anomaly. Journal of Memory and Language, 31, 785-806.

Murphy, E., \& Benítez-Burraco, A. (2019). Why Brain Oscillations are Improving Our Understanding of Language. Frontiers in Behavioral Neuroscience, 13, 190.

Patenaude, B., Smith, S. M., Kennedy, D. \& Jenkinson, M. (2011). A Bayesian model of shape and appearance for subcortical brain segmentation. NeuroImage, 56(3), pp. 907-922. http://doi.org/ 10.1016/j.neuroimage.2011.02.046

Perani, D., Saccuman, M. C., Scifo, P., Anwander, A., Spada, D., Baldoli, C., ... Friederici, A. D. (2011). Neural language networks at birth. Proceedings of the National Academy of Sciences, 108(38), 16056-16061. http://doi.org/10.1073/pnas.1102991108

Pinti, P., Tachtsidis, I., Hamilton, A., Hirsch, J., Aichelburg, C., Gilbert, S., \& Burgess, P. W. (2018). The present and future use of functional near-infrared spectroscopy (fNIRS) for cognitive neuroscience. Annals of the New York Academy of Sciences. http://doi.org/10.1111/nyas.13948

Pliatsikas, C. (2019). Understanding structural plasticity in the bilingual brain: The Dynamic Restructuring Model. Bilingualism: Language and Cognition, 1-13. https://doi.org/10.1017/S1366728919000130

Pliatsikas, C., DeLuca, V., Moschopoulou, E. \& Saddy, J. D. (2017) Immersive bilingualism reshapes the core of the brain. Brain Structure and Function, 222 (4). pp. 1785-1795. ISSN 1863-2661 doi: https://doi.org/10.1007/s00429-016-1307-9

Pliatsikas, C., Johnstone, T. \& Marinis, T. (2014) Grey matter volume in the cerebellum is related to processing of grammatical rules in a second language: a structural VoxelBased Morphometry study. The Cerebellum, 13 (1), pp. 55-63. doi: https://doi.org/10.1007/s12311-013-0515-6 
Pliatsikas, C., Moschopoulou, E., \& Saddy, J. D. (2015). The effects of bilingualism on the white matter structure of the brain. Proceedings of the National Academy of Sciences of the United States of America, 112(5), 1334-1337.

\section{http://doi.org/10.1073/pnas.1414183112}

Power, J. D., \& Schlaggar, B. L. (2017). Neural plasticity across the lifespan. Wiley Interdisciplinary Reviews Developmental Biology, 6(1), 10.1002/wdev.216. http://doi.org/10.1002/wdev.216

Prat, C. S., Yamasaki, B. L., \& Peterson, E. R. (2018). Individual differences in resting-state brain rhythms uniquely predict second language learning rate and willingness to communicate in adults. Journal of cognitive neuroscience, 31(1), 78-94.

\section{https://doi.org/10.1162/jocn_a_01337}

Prat, C. S., Yamasaki, B. L., Kluender, R. A., \& Stocco, A. (2016). Resting-state qEEG predicts rate of second language learning in adults. Brain and language, 157, 44-50.

\section{https://doi.org/10.1016/j.band1.2016.04.007}

Prystauka, Y., \& Lewis, A. G. (2019). The power of neural oscillations to inform sentence comprehension: A linguistic perspective. Language and Linguistics Compass, e12347.

Raichle, M.E., MacLeod, A.M., et al., (2001). A default mode of brain function. Proc. Natl. Acad. Sci. U. S. A. 98, 676-682. https://doi.org/10.1146/annurev-neuro-071013-014030

Raichle, M. E. \& Snyder, A. Z. (2007). A default mode of brain function: A brief history of an evolving idea. NeuroImage, 37, 1083-1090.

Rollans, C., Cheema, K., Georgiou, G. K., \& Cummine, J. (2017). Pathways of the inferior frontal occipital fasciculus in overt speech and reading. Neuroscience, 364, 93-106. http://doi.org/https://doi.org/10.1016/j.neuroscience.2017.09.011

Rossi, E., Kroll, J. F., \& Dussias, P. E. (2014). Clitic pronouns reveal the time course of 
procesing gender and number in a second langauge. Neuropsychologia, 62, 11-25.

Rossi, E. \& Prystauka, Y. (in press). Oscillatory brain dynamics of pronouns processing in native Spanish speakers and in late second language learners of Spanish. Bilingualism: Language and Cognition.

Ryan, C. (2013). Language Use in the United States: 2011. American Community Survey Reports, ACS-22. U.S. Census Bureau, Washington, DC. Retrieved from https://www.census.gov/library/publications/2013/acs/acs-22.html

Saito, K. (2017). Effects of sound, vocabulary, and grammar learning aptitude on adult second language speech attainment in foreign language classrooms. Language Learning, 67(3), 665-693. http://doi.org/10.1111/lang.12244

Sampaio-Baptista, C., \& Johansen-Berg, H. (2017). White matter plasticity in the adult brain. Neuron, 96(6), 1239-1251. http://doi.org/10.1016/j.neuron.2017.11.026

Silva, G., \& Citterio, A. (2017). Hemispheric asymmetries in dorsal language pathway whitematter tracts: A magnetic resonance imaging tractography and functional magnetic resonance imaging study. The Neuroradiology Journal, 30(5), 470-476. http://doi.org/10.1177/1971400917720829

Skeide, M. A., Brauer, J., \& Friederici, A. D. (2016). Brain functional and structural predictors of language performance. Cerebral Cortex, 26(5), 2127-2139. http://doi.org/10.1093/cercor/bhv042

Soares, J. M., Marques, P., Alves, V., \& Sousa, N. (2013). A hitchhiker's guide to diffusion tensor imaging. Frontiers in Neuroscience, March 12, 1-14. http://doi.org/10.3389/fnins.2013.00031

Statistics Canada. (August, 2017). Census in Brief: English-French bilingualism reaches new heights. Retrieved from https://www12.statcan.gc.ca/census-recensement/2016/as$\underline{\text { sa/98-200-x/2016009/98-200-x2016009-eng.cfm }}$ 
Surrain, S., \& Luk, G. (2019). Describing bilinguals: A systematic review of labels and descriptions used in the literature between 2005-2015. Bilingualism: Language and Cognition, 22(2), 401-415. doi:10.1017/S1366728917000682

Tolentino, L. C., \& Tokowicz, N. (2011). Across languages, space, and time: A review of the role of cross-language similarity in L2 (morpho) syntactic processing as revealed by fMRI and ERP methods. Studies in Second Language Acquisition, 33(1), 91-125. https://www.jstor.org/stable/44485982

Vaquero, L., Rodríguez-Fornells, A., \& Reiterer, S. M. (2017). The left, the better: Whitematter brain integrity predicts foreign language imitation ability. Cerebral Cortex, 27(8), 3906-3917. http://doi.org/10.1093/cercor/bhw199

Wan, N., Hancock, A. S., Moon, T. K., \& Gillam, R. B. (2018). A functional near-infrared spectroscopic investigation of speech production during reading. Human Brain Mapping, 39(3), 1428-1437. http://doi.org/10.1002/hbm.23932 